\section{SP0116 CASE 2 PRESENTER: BREAKING BAD AND TRAGICALLY HIP. A CASE OF A MISSED OPPORTUNITY AFTER VERTEBRAL FRACTURE}

Michael Skjødt. Norwegian University of Science and Technology (NTNU), Department of Public Health and Nursing, Trondheim, Norway

Mrs A is a 78-year old woman, admitted to the emergency room after a same level fall in her home earlier the same day. She has a history of Chronic Obstructive Pulmonary Disease with recurrent exacerbations, and hysterectomy at the age of 41 . At admittance, she is complaining of agonising pain localised to her right hip, and she is unable to move the leg. Upon the physical examination, the right leg is shortened and outwardly rotated. Upon further questioning, Mrs A reveals that she has lost four or five $\mathrm{cm}$ of height during the past years.

An X-ray of the hip demonstrates a neck of the femur fracture. Mrs A has surgery the following day and two screws are inserted. Due to a hospital-acquired pneumonia, Mrs A stays in the hospital for an additional 9 days after the operation, upon which she is discharged for a temporary placement at a nursing home for further rehabilitation.

Two months following discharge, Mrs A was called for a clinical evaluation by the hospital Fracture Liaison Service. Assessment with DXA confirms the osteoporosis diagnosis and VFA shows lower thoracic vertebral fractures. The FLS coordinator noted that Mrs A, related to a hospital admission for an acute exacerbation of her COPD 3 years before, had had a chest x-ray performed, on which vertebral fractures of the thoracic spine could be seen to be present. Mrs A has never previously been assessed nor treated for osteoporosis.

Disclosure of Interests: None declared

DOI: 10.1136/annrheumdis-2019-eular.8589

\section{SP0117 CASE 2 DISCUSSANT: AUTOMATED (IMPLEMENTED IN ELECTRONIC MEDICAL RECORD SYSTEMS) SHORT- TERM FRACTURE RISK PREDICTION}

Bo Abrahamsen. $x, x, x$, Denmark

Identification of patients at high risk of osteoporotic fractures is commonly based on fracture history, bone mineral density or a combination of the two. However, vertebral fractures are very often either missed or not acted upon by our health systems and DXA scanning to measure BMD in itself requires a coherent case finding strategy.

Current EPR systems may have built-in features to capture signs of sepsis or warnings to alert clinicians to cases with a diagnosis of atrial fibrillation with no apparent prescription for anti-coagulation. Building on the same principle, EPR systems or even national databases can be set up with appropriate decision rules to alert physicians to diagnoses such as RA, AS or COPD that we know strongly increases the risk of osteoporosis and to flag out medication patterns known to predict a high risk of osteoporotic fractures. This can then be checked against the presence of referrals for DXA and/or prescriptions for anti-osteoporosis medications and a clinical decision aid triggered to alert healthcare professionals. The mechanism powering such a tool can be an existing clinical risk algorithm such as qFracture, FRAX, FREM, Garvan FRC or others, depending on the nature of routinely collected data, the setting and the timescale required, or a bespoke risk tool developed for the healthcare system in question. It is important to appreciate that risk tools need careful calibration to be appropriate across countries and clinics and that the trigger risk level is ultimately a trade-off between clinical judgment and health economics.

Vertebral fractures represent a particular challenge. It has been established in the past that vertebral fractures often are not mentioned in radiology reports even if clearly present, or reported using unclear terminology that does not make it sufficiently clear to clinicians that a vertebral fracture is present. Moreover, even when vertebral fractures are called out in the radiology report it is not uncommon for this to go unnoticed. Opportunistic identification of vertebral fractures by software tools is a possible new avenue for narrowing the treatment gap in osteoporosis. With increasing integration of DICOM images into EPR systems, DICOMs of CT scans and other modalities containing spine images could be used either for automated case finding with alert boxes in the EPR system itself or employed in radiology departments to automatically populate reports with additional information about the presence of vertebral fractures.

\section{REFERENCES:}

[1] Rubin KH A New Fracture Risk Assessment Tool (FREM) Based on Public Health Registries. J Bone Miner Res. 2018 Nov;33(11):1967-1979.

[2] Reber KC Development of a risk assessment tool for osteoporotic fracture prevention: A claims data approach. Bone. 2018 May;110:170-176.

[3] Hippisley-Cox $\boldsymbol{J}$ Predicting risk of osteoporotic fracture in men and women in England and Wales: prospective derivation and validation of QFractureScores. BMJ. 2009 Nov 19;339:b4229.
[4] Moon RJ. Identification of patient profile for treatment. Best Pract Res Clin Endocrinol Metab. 2014 Dec;28(6):767-82.

[5] Rubin $\mathbf{K H}$ Risk assessment tools to identify women with increased risk of osteoporotic fracture: complexity or simplicity? A systematic review. J Bone Miner Res. 2013 Aug;28(8):1701-17.

Disclosure of Interests: None declared

DOI: 10.1136/annrheumdis-2019-eular.8412

FRIDAY, 14 JUNE 2019

13:30:00 - 15:00:00

\section{Primary and secondary fibromyalgia; are they different?}

\section{SP0118 FIBROMYALGIA; AN ACCEPTABLE DIAGNOSIS?}

Rinie Geenen. Utrecht University, Department of Psychology, Utrecht, Netherlands

Background: Neither patients nor clinicians and researchers fully embrace the fibromyalgia diagnosis. A core objection relates to the huge overlap with other polysymptomatic distress disorders such as somatic symptom disorder and chronic fatigue syndrome. Another objection has to do with the classification of fibromyalgia as a rheumatic disease, which may hamper research and treatment of psychosocial factors.

Objectives: To get insight into the pros and cons of the fibromyalgia diagnosis.

Methods: The scientific literature was reviewed with a focus on homogeneity and heterogeneity within the group diagnosed as fibromyalgia.

Results: That fibromyalgia is not a stable diagnosis of a homogeneous group is reflected in the ongoing development of new classification and diagnostic criteria; the newest including diagnostic criteria, common features, comorbidities, consequences, and putative mechanisms (Arnold et al., 2019). Moreover, treatment recommendations progressed from a focus on pharmacological treatment in 2008 to a focus on nonpharmacological therapies in 2017 (Macfarlane et al., 2017). Also, several studies showed that the group is quite heterogeneous with respect to type, number and severity of symptoms and comorbidities (e.g., Davis et al., 2018) as well as with respect to adaptation profiles covering a spectrum of severely maladjusted to quite well adjusted in terms of objective and subjective management (Estevez-Lopez et al., 2018).

Conclusion: Although diagnostic classifications such as fibromyalgia help to examine underlying mechanisms and interventions and to define treatment recommendations, in clinical practice and research account should be taken of heteroge neity of patients in terms of symptoms and disorder-transcending biopsychosocial factors that influence the disorder. This suggests that fibromyalgia is an acceptable diagnosis to the extent that individuality is accepted as part of the diagnosis.

\section{REFERENCES:}

[1] Arnold, et al. AAPT Diagnostic Criteria for Fibromyalgia. J Pain 2019, in press.

[2] Davis, et al. Characterizing classes of fibromyalgia within the continuum of central sensitization syndrome. J Pain Res 2018;11:2551-60.

[3] Estevez-Lopez, et al. Adaptation profiles comprising objective and subjective measures in fibromyalgia: the al-Andalus project. Rheumatology (Oxford) 2017:56:2015-24

[4] Macfarlane, et al. EULAR revised recommendations for the management of fibromyalgia. Ann Rheum Dis 2017;76:318-28.

Disclosure of Interests: None declared DOI: 10.1136/annrheumdis-2019-eular.8484

\section{SP0119 SEARCHING FOR PATHOLOGY; HOW HARD SHOULD WE LOOK?}

\section{Ernest Choy. CREATE Centre, United Kingdom}

Background: The 1990 American College of Rheumatology (ACR) classification criteria for Fibromyalgia (FM) was intended to facilitate research by defining a homogenous patient population [1]. Two key features of the criteria are tender point examination and exclusion of other conditions that may cause widespread pain. This led to the view that FM is a diagnosis of exclusion. This is no longer the case in 2019.

Results: In 2010, the ACR published provisional criteria for the diagnosis of FM [2]. These allowed FM to be diagnosed by using questionnaire removing the need for tender point examination. One of the major advantages of the criteria is that the diagnosis of FM can be made by primary care physician and the first-line treatment be initiated without referral to secondary care. Such an approach was 